\title{
CONTRIBUTION TO A KNOWLEDGE OF THE MOSSES OF NEW SOUTH WALES.
}

\section{By William Forsyth.}

If an apology were required for bringing this paper under the notice of the members of this Society, the general want of knowledge prevailing even amongst botanists with respect to the mosses of New South Wales would, I think, be considered ample.

No list or catalogue of the mosses of Australia has been published since the date of issue of Mr. Mitten's list in 1882. Many species have been discovered since that time, but from the fact that nearly all the collections have been sent for determination to specialists in Europe, and the descriptions of new species published in European publications - not always obtainable here -information regarding them is very meagre indeed, and to ascertain what has or has not been recorded is a matter of no little difficulty and some uncertainty.

It is, therefore, with the object of furnishing some little information with regard to geographical distribution, etc., that the writer submits the following list. Many of the species have not hitherto been recorded for New South Wales, and some of them have been recorded in so indefinite a manner as to be next to useless so far as affording any guide to the collector is concerned. Some new localities also are given for species already definitely recorded.

The total number of species under notice is 61 , of which 43 are new for the colony, the remaining number furnishing new localities; and 26 are recorded from Port Jackson.

Although much care has been taken to have the given localities, etc., as full and correct as possible, still some incompleteness cannot be unexpected in a list which must neccessarily be of a tentative character.

It has only to be stated that the specimens from which the species were determined passed through the hands of the eminent bryologist, Dr. Brotherus, of Helsingfors, to banish any doubt as to the correctness of the naming. 
I have to acknowledge my indebtedness to Mr. R. A. Bastow, F.L.S., well known for his work on Tasmanian Mosses, for a copy of his MS. "List of Australian Mosses." The localities marked with an asterisk are from this as yet unpublished list. I have also to thank Mr. T. Whitelegge and Rev. W. W. Watts for the opportunity of using some of their records.

No previous records for species are given outside of Australia and Tasmania.

Tribe i. DICRANEÆ, Mitten.

D I C R A N E L L A, C.M.

Dicranella Dietrichie, C.M.

National Park (W. Forsyth; Sept. 13th, 1898); Fitzroy Falls (T. Whitelegge; Nov. 8th, 1884).

Previously recorded from Queensland and New South Wales.*

Dicranella tricruris, C.M.

Turramurra, near Sydney (W. Forsyth; Aug. 14th, 1898) and Burringbar; Nov. 11th, 1898); Richmond River (Rev. W. W. Watts; April 29th, 1896); Gosford (T. Whitelegge; Sept., 1891).

Previously recorded from Queensland.

B L I N D I A, Bruch \& Schimper.

Blindia robusta, Hampe.

Merritt's Camp, Kosciusko (W. Forsyth; Jan., 1899).

Previously recorded from Tasmania* and Victoria.

This handsome moss was found growing just a few feet from the edge of a large snow drift, and in some instances partly covered by the melting snow.

\section{H O L O M I T R I U M.}

Holomitrium perichetiale, Bridel.

Lane Cove, Port Jackson (W. Forsyth; July, 1898); Ballina, Alstonville Road (Rev. W. W. Watts; Dec., 1897).

Previously recorded from Tasmania, Victoria and Queensland.* 
Ex с а м т o d o n, Montague.

Encamptodon Muelleri, Hpe. \& C.M.

Mt. Warning (W. Forsyth; Oct. 31st, 1898); Brooklet, Richmond River (Rev. W. W. Watts; Sept., 1896).

Previously recorded from Victoria.

Ca m P y o P U s, Bridel.

Campylopus Woollsi, C.M.

Top of Mt. Warning, growing on the stems of Xanthorrhoea arborea, R.Br. (31st. Oct., 1898), National Park (July, 1898), Three Mile Scrub, Byron Bay (7th Nov., 1898), and Turramurra (W. Forsyth; 14th Aug., 1898); Wyong (A. A. Hamilton; April, 1899).

Previously recorded by the late Rev. Dr. Woolls from Sydney, but without any definite locality; also recorded from Queensland.

\section{Tribe ii. GRIMMIEA.}

G R I M M I A, Ehrhart.

Grimmia apocarpa, Hedwig.

Merritt's Camp, Kosciusko; growing plentifully on the rocks above Merritt's Camp, on the Kosciusko Plateau (W. Forsyth; Jan., 1899).

Previously recorded from Tasmania and Victoria.

Grimmia trichophylla, Grev.

Pretty Point, Kosciusko (W. Forsyth; Jan., 1899).

Previously recorded from Tasmania and Queensland.*

Grimmia cygnicolla, Tayl., (G. pulvinata, Hook. \& Tayl.)

Barber's Creek (J. H. Maiden; Oct., 1898).

Previously recorded from Western Australia, Tasmania, Victoria and Queensland.* 
Grimma obtusata, Hpe. \& C.M.

Merritt's Camp, Kosciusko (W. Forsyth; Jan., 1899).

Previously recorded from Victoria.*

G L у р н о м I т R I U м, Bridel.

Glyphomitrium Muelleri, Mitten.

Bowmark (J. H. Maiden; Sept. 14th, 1897).

Previously recorded from Queensland and New South Wales.*

Tribe iii. LEUCOBRYEE.

L E U C O в R Y U i, Hampe.

Leucobryum strictifolium, Broth.

Lawson, Blue Mts. (E. Betche; Aug., 1895); Cowan Creek (A. A. Hamilton; Jan., 1899).

Previously recorded from Queensland; and Richmond River, New South Wales (Rev. W. W. Watts).

Tribe v. TORTULEE.

W e i s s I A, Hedwig.

Weissia flavipes, J. Hook. \& Wils.

La Perouse, Botany Bay (W. Forsyth; July, 1899); Richmond River (Rev. W. W. Watts).

Previously recorded from Tasmania and Victoria.

H y м Е N O $\mathrm{S}$ т о м $\mathrm{U}$ M, R.Br.

Hymenostonum olivaceum, C.M.

Nepean River (W. Forsyth; Sept. 23rd, 1898).

Previously recorded from Gosford (T. Whitelegge, 1891).

T O R T U L A, Hedwig.

Tortula PRInceps, DeNotaris.

Jenolan (J. H. Maiden; Aug., 1898).

Previously recorded from Tasmania* and Victoria. 
Tortula (Barbula) subcalycina, C.M.

Lane Cove (W. Forsyth; July, 1898); Rookwood (E. Cheel; Aug. 7th, 1898).

Previously recorded from Queensland.*

\section{Tortula calycina, C.M.}

National Park (W. Forsyth; Sept. 10th, 1898).

Previously recorded from West Australia, Tasmania and Queensland.

E U c a l y t A, Schreber.

Eucalypta tasmanica, Hpe. \& C.M.

Jenolan (J. H. Maiden; Aug., 1898); Shoalhaven Gullies, near Badgery's Crossing (W. Forsyth; Sept., 1899); and Warrumbungle Ranges (Oct., 1899).

Previously recorded from Tasmania.

Tribe vi. ORTHOTRICHEE.

O R т H O D o t i u m, Schreber.

Orthodontium lineare, 'laylor.

La Perouse (W. Forsyth; July, 1898); Gosford (T. Whitelegge; Sept. 20th, 1891).

Growing abundantly on the lower part of the trunks of Eucalyptus robusta at La Perouse, Botany Bay. I have a suspicion that plants of this species with mature fructification form the $O$. ovale of C.M.

Previously recorded from West Australia* and Tasmania.

Orthodontium sulcatum, Hook.

Tempe, near Sydney (A. A. Hamilton; Sept. 18th, 1898).

Previously recorded from Victoria.

$$
\text { Orthodontium ovale (?) C.M. }
$$

La Perouse (W. Forsyth; Dec., 1898).

Previously recorded from Gosford, New South Wales ( $T$. Whitelegge, 1891). 
This moss also grows plentifully on the trunks of trees of Eucalyptus robusta at La Perouse.

Tribe vii. SPLACHNEE.

T A Y L O R I A, Hook.

TaYloria octoblepharis, Hook.

Turramurra (Aug., 1898), Lane Cove (July, 1898), Nepean River (Sept., 1898), and National Park (W. Forsyth; Aug., 1898); Appin (J. H. Maiden; Sept., 1898); Mosman's Bay (T. Whitelegge, 1884).

Strange as it may appear I cannot find any record of this moss for New South Wales. It is a common moss, and I am aware that it has been found by Mr. Whitelegge, Mr. Watts and others.

Tribe viii. FUNARIEÆ.

F U N A R I A, Schreber.

Funaria aristata, Broth.

Lane Cove (W. Forsyth; July, 1ø98, and Sept., 1898).

Previously recorded from Lilyvale, New South Wales ( $T$. Whitelegge; Sept., 1891).

Funaria cuspidata, Hook. \& Wils.

La Perouse (W. Forsyth; July, 1898).

Previously recorded from Victoria. *

Funaria glabra, Tayl.

Turramurra (W. Forsyth; 14th Aug., 1898), Lane Cove (Sept., 1898), and National Park (10th Sept., 1898).

Previously recorded from West Australia, Tasmania and Victoria.

\section{Tribe ix. BARTRAMIEE.}

Ph i l o n o t is, Bridel.

Philonotis appressa, Hook. \& Wils.

Mt. Kosciusko (W. Forsyth; Jan., 1899).

Previously recorded from Tasmania and Victoria. 
Con os т о U M, Swartz.

Conostomum pusillum, Hook. \& Wils.

King's Tableland, Blue Mts. (W. Forsyth; Nov. 2nd, 1898), and Mt. Kosciusko (Jan., 1899).

Previously recorded from Tasmania and Victoria.

The specimens from Mt. Kosciusko are much more robust than those from King's Tableland, their general appearance being very different.

Conostonum curvirostre, Mitten.

Summit of Mt. Kosciusko (W. Forsyth; Jan., 1899).

We found this quaint little moss growing on the summit of Kosciusko close to the Observatory.

Previously recorded from Victoria.

B A R T R A M I A, Hedwig.

Bartramia papillata, Hook. \& Wils.

Merritt's Camp, Kosciusko (W. Forsyth; Jan., 1899).

Previously recorded from Tasmania, Victoria and Queensland.* Tribe $x$. BRYEE.

B R Y U M, Linn.

Bryum leptothecium, Tayl.

Jenolan Caves (H. Malthouse; Aug., 1898); Manly (A. A. Hamilton; Sept., 1898).

Previously recorded from Tasmania and Victoria.

Bryum creberrimum, Tayl.

Lane Cove (W. Forsyth; July, 1898).

Previously recorded from West Australia.

\section{Bryum pachytheca, C.M.}

"Thirteen miles from Dubbo" (J. H. Maiden; Aug. 8th, 1898); Rookwood (E. Cheel; Aug. 7th, 1898). 
Previously recorded from West Australia, Victoria and Tasmania.

Mitten in his list put this species and B. baloides, Tayl., underthe head of $B$. dichotomum, Hedw., but the Index Bryologicus keeps the species (pachytheca) distinct. As Mr. Mitten gives the three colonies-West Australia, Victoria and Tasmania-for the three so-called species under the heading of dichotomum, it would be interesting to know how many of the colonies have recorded "pachytheca."

Bryum chrysoneuron, C.M.

Lane Cove (W. Forsyth; July, 1898).

Previously recorded from Tasmania and Victoria.

Bryum pyrothecium, Hpe. \& C.M.

King's Tableland (W. Forsyth; Oct., 1898).

Previously recorded from Victoria.

Bryum erythropyxis, C.M.

National Park (W. Forsyth; Aug., 1898), and La Perouse (July, 1898).

Previously recorded from Hume River (Miss Campbell, 1881); Cambewarta (T. Whitelegge, 1881).

\section{Bryum (Webera) nutans.}

Merritt's Camp, Kosciusko (W. Forsyth; Jan., 1899).

Previously recorded from Tasmania, Victoria and New South Wales.*

R н I z o g o N I $\mathrm{m}$, Bridel.

Rhizogonium Nove-Hollandie, Bridel.

Burringbar (W. Forsyth; Nov. 5th, 1898).

Previously recorded from Tasmania and Victoria. 
Tribe xii. RHACOPILEAE.

R н A с O P I L U H, Bridel.

Rhacopilum convolutaceum, C.M.

Jenolan Caves (H. Malthouse; Aug., 1898); King's Tableland (W. Forsyth; Oct., 1898); Mosman's Bay ( $T$. Whitelegge; Sept., 1884).

Previously recorded from Richmond River (Camara); also from Tasmania and Queensland.

\section{Tribe xv. NECKEREA.}

L E P T o D o N, Mohr.

Leptodon Smithii, Mohr.

Mt. Tomah (J. H. Maiden; Nov., 1898); Shoalhaven Gullies (W. Forsyth; Sept., 1899).

Previously recorded from New South Wales, but no locality given.

Tribe xvi. SEMATOPHYLLEA.

R H A P H D O T E G I U, Schreber.

Rhaphidostegium acicula, C.M.

National Park (W. Forsyth; July, 1898), La Perouse (July, 1898); Wyong (A. A. Hamilton; April, 1899); Cook's River (Rev. W. W. Watts; March, 1896); Lord Howe Island (J. H. Maiden; April, 1898).

Previously recorded from Victoria.*

\section{Rhaphidostegium Luciduloides, C.M.}

Lane Cove (W. Forsyth; July, 1898), National Park (Aug., 1898), Cooper Estate, Woollahra (Aug., 1898); “Near Gordon” (Rev. W. W. Watts; Oct., 1895).

Previously recorded from New South Wales, * but no locality given. 
Rhaphidostegium pseudo-homonallum, C.M.

Murwillumbah ( $W$. Forsyth; Nov. 3rd, 1,898); Richmond River (Rev. W. W. Watts; July 7th, 1898); Wyong (A. A. Hamilton; April 4th, 1898).

Previously recorded from New South Wales (Homebush; T. Whitelegge, 1885).

A c a t h o C L a d i u M, Mitten.

Acanthocladium extenuatum, Bridel.

Turramurra (W. Forsyth; Aug. 14th, 1898); Jenolan Caves (H. Malthouse; Aug., 1898).

Previously recorded from Tasmania, Victoria, Queensland*; and New South Wales) Macquarie River (Ball).

Tribe xvii. STEREODONTEE.

I S O P T E R Y G I U M, Mitten.

Isopterygium Candidun, C.M.

Mosman's Bay (W. Forsyth; Sept. 17th, 1898); Richmond River (Rev. W. W. Watts; Jan. 5th, 1897).

Previously recorded from "Near Sydney" (Kayser) and Queensland.*

I have thought it desirable to record these localities, thus making it definite.

\section{Isopterygium viridi-Pallidus, C.M.}

Cooper Estate, Woollahra (W. Forsyth; Aug., 1898); La Perouse (July, 1898); Wyong (A. A. Hamilton; April, 1899); Richmond River (Rev. W. W. Watts).

I can find no previous record.

E c t r о р о т н е с I M, Mitten.

Ectropothecium umbilicatum, C.M.

Ballina (W. Forsyth; Oct., 1898); Richmond River (Rev. W. W. Watts; May 2nd, 1896). 
I cannot find any previous record of this species; probably Queensland.

Tribe xviii. HYPNEA.

F A B R O N A A, Raddi.

Fabronia Scottiæ, C.M.

Como (E. Cheel; Nov., 1898); Richmond River, Tentenbar Road (Rev. W. W. Watts; Nov., 1896).

Previously recorded from Queensland* and New South Wales (Hunter River; Mrs. Ford).

R H Y N CH O S TE G I U M, Schimper.

Rhynchostegium tenufolium, Hedwig.

National Park (W. Forsyth; Aug, 1898); King's Tableland (Oct., 1898).

Previously recorded from Victoria.

B r A C h т t h e i U m, Schimper.

Brachythecium (Hypnum) paradoxum, Hook. \& Wils.

Merritt's Camp, Kosciusko (W. Forsyth; Jan., 1899).

Previously recorded from Tasmania and Victoria.

T h U I D I U, Schimper.

Thuidium furfurosum, Hook. \& Wils.

National Park (W. Forsyth; July, 1898); Jenolan (H. Malthouşe; Aug., 1898).

Previously recorded from Tasmania and Victoria.

Thuidium protensula, C.M.

Burringbar (W. Forsyth; Nov. 5th, 1898).

I cannot find any record of this species. 
Tribe xix. SKITOPHYLLE⿸厂

F I s s I D E N s, Bridel.

Fissidens tenellus, Hook. \& Wils.

Lane Cove (W. Forsyth; Aug., 1898); Richmond River (Rev. W. W. Watts; June 22nd, 1896).

Previously recorded from Tasmania and Victoria.

Fissidens pallidus, Hook. \& Wils.

Mt. Tomah (J. H. Maiden; Nov., 1898); National Park (W. Forsyth; July, 1898); Richmond River (Rev. W. W. Watts; Aug. 4th, 1896); Lawson (E. Betche; Aug., 1895).

Previously recorded from Tasmania.

\section{Fissidens coarctatus, C.M.}

National Park (W. Forsyth; July, 1898); Richmond River (Rev. W. W. Watts; June 29th, 1896, and Aug. 1st, 1896).

Previously recorded from New South Wales.*

Fissidens liliputano-incurvus, C.M.

Lane Cove (W. Forsyth; July, 1898), National Park (July, 1898).

Previously recorded from Victoria.*

Fissidens semilimbatus, Hpe. \& C.M.

Lane Cove (W. Forsyth; Sept., 1898).

Previously recorded from Tasmania* and Victoria.

Fissidens incurvo-bryoides, C.M.

Rookwood (E. Cheel; Aug. 7th, 1898).

I cannot find any record of this species.

Tribe $\mathrm{xx}$. POLYTRICHEA.

P s i l o P I L U m, Bridel.

Psilopilum australe, Hook. \& Wils.

Mt. Kosciusko (W. Forsyth; Jan., 1899).

Previously recorded from Tasmania. 
Pog on t U m, Bridel.

Pogonatum alpinum, Linn.

Mt. Kosciusko (W. Forsyth; Jan., 1899).

Previously recorded from Tasmania and Victoria.

Pogonatum australasicun, Hpe. \& C.M.

National Park (W. Forsyth; 10th Sept., 1898), Burringbar (Nov. 5th, 1898); Lawson (A. A. Hamilton; Oct. 15th, 1898).

Previously recorded from Victoria.*

P o l y t R I C H U m, Dillenius.

Polytrichum juniperinum, Willd.

Mt. Kosciusko (W. Forsyth; Jan., 1899).

Previously recorded from Tasmania and Victoria. 


\section{$2 \mathrm{BHL}$ Biodiversity Heritage Library}

Forsyth, W. 1900. "Contribution to a knowledge of the mosses of New South Wales." Proceedings of the Linnean Society of New South Wales 24, 674-686. https://doi.org/10.5962/bhl.part.7690.

View This Item Online: https://www.biodiversitylibrary.org/item/21735

DOI: https://doi.org/10.5962/bhl.part.7690

Permalink: https://www.biodiversitylibrary.org/partpdf/7690

\section{Holding Institution}

MBLWHOI Library

\section{Sponsored by}

MBLWHOI Library

\section{Copyright \& Reuse}

Copyright Status: NOT_IN_COPYRIGHT

This document was created from content at the Biodiversity Heritage Library, the world's largest open access digital library for biodiversity literature and archives. Visit BHL at https://www.biodiversitylibrary.org. 